\title{
Sparsity-based Algorithm for Detecting Faults in Rotating Machines*
}

\author{
Wangpeng $\mathrm{He}^{\dagger 1,2}$, Yin Ding ${ }^{\ddagger 1}$, Yanyang $\mathrm{Zi}^{2}$, and Ivan W. Selesnick ${ }^{1}$ \\ ${ }^{1}$ Tandon School of Engineering, New York University, 6 Metrotech Center, Brooklyn, NY 11201, USA \\ ${ }^{2}$ State Key Laboratory for Manufacturing and Systems Engineering, School of Mechanical Engineering, Xi'an \\ Jiaotong University, Xi'an 710049, P. R. China
}

August 23, 2018

\begin{abstract}
This paper addresses the detection of periodic transients in vibration signals for detecting faults in rotating machines. For this purpose, we present a method to estimate periodic-group-sparse signals in noise. The method is based on the formulation of a convex optimization problem. A fast iterative algorithm is given for its solution. A simulated signal is formulated to verify the performance of the proposed approach for periodic feature extraction. The detection performance of comparative methods is compared with that of the proposed approach via RMSE values and receiver operating characteristic (ROC) curves. Finally, the proposed approach is applied to compound faults diagnosis of motor bearings. The non-stationary vibration data were acquired from a SpectraQuest's machinery fault simulator. The processed results show the proposed approach can effectively detect and extract the useful features of bearing outer race and inner race defect.
\end{abstract}

\section{Introduction}

Rotating machinery is one of the most common types of mechanical equipment and plays a significant role in industrial applications. Early detection of faults developing in rotating machinery is of great importance to prevent economic loss and personal casualties 11. Rolling element bearings and gearboxes are two kinds of widely used components in rotating machines and their failures are among the most frequent reasons for machine breakdown.

Much attention has focused on vibration-based diagnosis of mechanical faults in rotating machines [2]. The detection of periodically occurring transient vibration signatures is of vital importance for vibrationbased condition monitoring and fault detection of rotating machinery 3. However, these useful transient features are usually buried in heavy background noise and other irrelevant vibrations. To address this problem, many signal processing methods have been introduced, such as singular value decomposition (SVD) [4], empirical mode decomposition (EMD) [5], and methods based on different wavelet transforms, e.g., dualtree wavelet in [6], harmonic wavelet in [7, and tunable Q-factor wavelet (TQWT) in [8]. These methods have achieved successful applications in the field of machinery fault diagnosis.

Recently, an algorithm, called 'overlapping group shrinkage' (OGS) was developed for estimating groupsparse signals in noise 9 . The OGS algorithm was initially formulated as a convex optimization promoting

\footnotetext{
*Preprint submitted to Mechanical Systems and Signal Processing (Elsevier).

†Email: wangp.he@gmail.com

‡Email: yd372@nyu.edu
} 
group sparsity by a convex regularization. In order to promote sparsity more strongly, an improved version of OGS was developed, which utilizes a non-convex regularization [10. The superiority of denoising groupsparse signals using the approach presented in [10] indicates its potential for effectively extracting periodic transient pulses.

This paper aims to develop an approach for rotating machinery fault diagnosis based on a periodic groupsparse signal representation. The signature of localized faults of the gear teeth and bearing components generally exhibit periodic transient pulses when a rotating machine is operated at a constant speed [11]. Meanwhile, the large amplitudes of these useful features are not only sparse but also tend to form groups. Several neighborhood-based denoising methods have been developed for machinery fault diagnosis utilizing this property 12 14]. Our approach is based on a signal model intended to capture the useful impulsive features for machinery fault diagnosis. In particular, this paper addresses the problem of estimating $x$ from a noisy observation $y$. We model the measured discrete-time series, $y$, as

$$
y_{n}=x_{n}+w_{n}, \quad n=0, \ldots, N-1,
$$

where the signal $x$ is known to have a periodic group-sparse property and $w$ is white Gaussian noise. A group-sparse signal is one where large magnitude signal values tend not to be isolated. Instead, these large magnitude values tend to form groups.

Convex optimization is commonly used to estimate sparse vectors from noisy signals, where we solve the optimization problem with the prototype

$$
x^{*}=\arg \min _{x}\left\{F(x)=\frac{1}{2}\|y-x\|_{2}^{2}+\lambda \Phi(x)\right\},
$$

where $\lambda>0$ is a regularization parameter and $\Phi: \mathbb{R}^{N} \rightarrow \mathbb{R}$ is a sparsity-promoting penalty function (regularizer). Conventionally, the regularizer $\Phi(x)$ is a convex function, e.g. $\ell_{1}$-norm. In [15, an idea of using non-convex regularizer and keeping the convexity of entire problem is used for signal denoising problem, where the sparsity can be significantly promoted comparing to $\ell_{1}$-norm, and the problem still has a unique solution due to the convexity.

In this paper, we adopt ideas from [15] and 9], and present a method for estimating periodic-group-sparse signals in noise. We propose its use for detecting faults in rotating machinery, where the fault characteristic frequency (period of the group-sparse pulses) is used as prior knowledge. Similar to the approach in [10], the non-convex regularization term in the proposed method is properly chosen so as to ensure that the total objective function $F$ is convex; however, in contrast to [10, where each group has to be contiguous, we allow grouping with intervals, and furthermore periodically.

As a consequence, in this work, the regularization term $\Phi$ in 2 is formulated specifically to utilize the periodicity of the impulsive fault features. The aim of our approach is to capture the useful impulsive features for the purpose of machinery fault diagnosis. Additionally, it has the potential to separate compound fault features by utilizing different periods of the periodic transient pulses corresponding to different fault frequencies (e.g., various defect frequencies of rolling element bearings). The proposed approach also reduces to a non-periodic group-sparse signal denoising method, i.e., we can utilize the sparsity-based OGS approach [10], if we do not have prior knowledge of the period of the group-sparse transients. Thus, the proposed sparsity-based approach is a generalization of the non-convex regularized OGS 10.

The paper is organized as follows. A brief review of OGS with convex and non-convex regularization is given in Section 2. Section 3 presents a method for denoising periodic group sparse signals. In Section 4 a simulation study is performed to validate the effectiveness of the proposed method. Section 5 applies the 
proposed periodic group sparse denoising method to fault diagnosis of motor bearings for further validation of its effectiveness. Finally, conclusions are summarized in Section 6.

\section{Review}

In this section, we give short reviews of overlapping group shrinkage (OGS) 9 and majorization-minimization (MM) [16].

\subsection{Overlapping Group Shrinkage (OGS)}

There are several advantages to formulating sparse estimation as a convex optimization problem. The most basic advantage is that the problem can then be very reliably and efficiently solved using convex optimization methods 17. Although a non-convex regularizer can promote sparsity more strongly, it generally leads to a non-convex optimization problem with non-optimal local minima 18. To avoid the formulation of a nonconvex optimization problem, one may utilize a non-convex regularizer $\Phi$ designed so as to ensure the total objective function is convex.

The problem of denoising a group sparse signal was addressed in [9] which utilized convex optimization. An improved method was proposed in [10, which utilized non-convex regularization designed to ensure convexity of the objective function. The problem is solved efficiently by an iterative algorithm based on majorization-minimization (MM) [16. The objective function for the OGS problem, with a group size of $K$ and convex or non-convex regularization, is denoted as

$$
x^{*}=\arg \min _{x \in \mathbb{R}^{N}}\left\{P_{0}(x)=\frac{1}{2}\|y-x\|_{2}^{2}+\lambda \sum_{n} \phi\left(\left[\sum_{k \in \mathcal{K}} x_{n+k}^{2}\right]^{1 / 2} ; a\right)\right\}
$$

where $\mathcal{K}:=\{0,1, \ldots K-1\}$, and $y \in \mathbb{R}^{N}$ is the noisy observation. For $x \in \mathbb{R}^{N}$, we define $x_{n}=0$ for $n<0$ and $n \geqslant N$. We assume the penalty function $\phi: \mathbb{R} \rightarrow \mathbb{R}_{+}$satisfies the properties:

1. $\phi$ is continuous on $\mathbb{R}$.

2. $\phi$ is twice differentiable on $\mathbb{R} \backslash\{0\}$.

3. $\phi$ is increasing and concave on $\mathbb{R}_{+}$.

4. $\phi$ is symmetric, $\phi(-x ; a)=\phi(x ; a)$.

5. $\phi^{\prime}\left(0^{+} ; a\right)=1$.

6. $\phi^{\prime \prime}(x ; a) \geqslant-a$ for all $x \neq 0$.

7. $\phi(x ; 0)=|x|$.

which is used to induce the resulting sparsity in an optimization problem.

Note that the objective function $P_{0}$ in (3) may be convex even if the regularizer (penalty) is not. When the penalty function $\phi$ satisfies the conditions listed above, then the parameter ' $a$ ' can be chosen so that $P_{0}$ is convex even if $\phi$ is not [15. This approach is used to improve OGS in 10] where it has been proved that the objective function $P_{0}$ in $(3)$ is strictly convex if

$$
0 \leqslant a<\frac{1}{\lambda K} .
$$




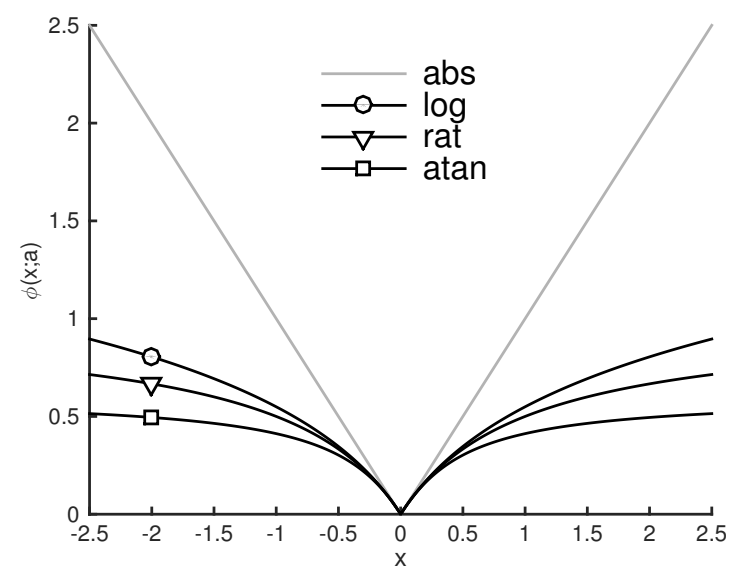

Figure 1: Examples of penalty functions.

Table 1: Sparsity-promoting penalty functions $(a \geqslant 0)$.

\begin{tabular}{lll}
\hline Penalty & $\phi(x ; a)$ & $\psi(x)$ \\
\hline abs & $|x|$ & $|x|$ \\
$\log$ & $\frac{1}{a} \log (1+a|x|)$ & $|x|(1+a|x|)$ \\
rat & $\frac{|x|}{1+a|x| / 2}$ & $|x|(1+a|x|)^{2}$ \\
atan & $\frac{2}{a \sqrt{3}}\left(\tan ^{-1}\left(\frac{1+2 a|x|}{\sqrt{3}}\right)-\frac{\pi}{6}\right)$ & $|x|\left(1+a|x|+a^{2}|x|^{2}\right)$ \\
\hline
\end{tabular}

Table 1 gives some examples of penalty functions satisfying the above-listed conditions, and these examples are illustrated in Fig. 1. In order to induce the sparsity most strongly, the arctangent 'atan' function can be used among all the three given non-convex functions. Fig. 1 1 also illustrates that under the same ' $a$ ' parameter, 'atan' function adheres the most 'concavity'. In addition to these non-convex penalty functions, Fig. 1 also shows the $\ell_{1}$-norm as a special case. Note that we allow $a=0$, which is the extreme case of the three other penalties. In particular, $\phi(x ; 0)=|x|$ is a convex function. The original OGS algorithm given in [9] considers only the special case $a=0$, i.e., convex regularization.

\subsection{Majorization-minimization (MM)}

A detailed derivation to solve problem (3), based on the majorization-minimization (MM) method is given in 10. The MM method simplifies a complicated optimization problem into a sequence of easy ones, and is described by the iteration

$$
u^{(i+1)}=\arg \min _{u} G\left(u, u^{(i)}\right)
$$


where $i$ denotes the number of iterations, and $G: \mathbb{R}^{N} \times \mathbb{R}^{N} \rightarrow \mathbb{R}$ is a majorizer of the objective function $J$, satisfying

$$
\begin{aligned}
& G(u, v) \geqslant J(u), \quad \text { for all } u, v, \\
& G(u, u)=J(u) .
\end{aligned}
$$

A majorizer of $\phi$ is given by

$$
g(u, v)=\frac{\left(u^{2}-v^{2}\right)}{2 \psi(v)}+\phi(v ; a), \quad \text { when } v \neq 0
$$

where $\psi$ is the function given in Table 1 . For $v=0, g(u, 0)$ is defined by

$$
g(u, 0):= \begin{cases}+\infty, & \text { if } u \neq 0 \\ 0, & \text { if } u=0\end{cases}
$$

As a consequence, the function $g: \mathbb{R} \times \mathbb{R} \rightarrow \mathbb{R}_{+}$satisfies

$$
\begin{aligned}
& g(u, v) \geqslant \phi(u ; a), \quad \text { when } u \neq v, \\
& g(u, u)=\phi(u ; a),
\end{aligned}
$$

Note that $g(u, 0)$ defined in (8) equals infinity except $u=0$. This forces its minimizer to lock to $u=0$ in the MM iteration described in (5). This issue in the OGS problem is discussed in 10, where it does not affect the convergence when the algorithm is implemented with a non-zero initialization.

\section{OGS with binary weights}

To facilitate the following derivation, we define a binary sequence $b=\left[b_{0}, b_{1}, \ldots b_{K-1}\right]$, with $b_{k} \in\{0,1\}$, and sets

$$
\begin{aligned}
& \mathcal{K}:=\{0,1, \ldots, K-1\} \\
& \mathcal{K}_{0}:=\left\{k \in \mathcal{K}: b_{k}=0\right\}, \\
& \mathcal{K}_{1}:=\left\{k \in \mathcal{K}: b_{k}=1\right\}
\end{aligned}
$$

Since $b$ is a binary vector, we have $\mathcal{K}=\mathcal{K}_{0} \cup \mathcal{K}_{1}$ and $\mathcal{K}_{0} \cap \mathcal{K}_{1}=\emptyset$ is the empty set. We denote the cardinality (size) of the sets $\mathcal{K}, \mathcal{K}_{1}$ and $\mathcal{K}_{0}$ as $K, K_{1}$, and $K_{0}$, respectively, so that $K=K_{0}+K_{1}$, and

$$
\sum_{k \in \mathcal{K}} b_{k}=\sum_{k \in \mathcal{K}_{1}} b_{k}=K_{1}
$$

The following proposition is straightforward.

Proposition 1. Let $\phi: \mathbb{R} \rightarrow \mathbb{R}$ satisfy the conditions listed in Section 2 . When $\gamma>0$ and $\lambda>0$, the function $p: \mathbb{R} \rightarrow \mathbb{R}$,

$$
p(v)=\frac{\gamma}{2} v^{2}+\lambda \phi(v a)
$$


is strictly convex if

$$
\phi^{\prime \prime}(v ; a)>-\frac{\gamma}{\lambda} .
$$

Detailed proof of this proposition can be found in Appendix A

\subsection{Problem definition}

We define the objective function $P_{1}: \mathbb{R}^{N} \rightarrow \mathbb{R}$ as

$$
x^{*}=\arg \min _{x}\left\{P_{1}(x)=\frac{1}{2}\|y-x\|_{2}^{2}+\lambda \sum_{n} \phi(\theta(x, b, n) ; a)\right\}
$$

where the binary-weighted grouping function $\theta: \mathbb{R}^{N} \times \mathbb{R}^{K} \times \mathbb{Z} \rightarrow \mathbb{R}$ is defined as

$$
\theta(x, b, n):=\left[\sum_{k=0}^{K-1} b_{k} x_{n+k}^{2}\right]^{1 / 2}
$$

which is the Euclidean norm of a binary weighted block. For $x \in \mathbb{R}^{N}$, we define $B_{n}(x) \in \mathbb{R}^{K}$ as

$$
B_{n}(x):=\left[x_{n}, x_{n+1}, \ldots x_{n+K-1}\right],
$$

i.e., a $K$-point subvector of $x$, starting at index $n$. Hence $\theta(x, b, n)$ can be written as

$$
\theta(x, b, n)=\left\|b \odot B_{n}(x)\right\|_{2},
$$

where $\odot$ denotes element-wise multiplication.

Note that in (14) if $b_{k}=1$ for all $k \in \mathcal{K}$, then $\theta^{2}(x, b, n)=\sum_{k} x_{n+k}^{2}$, and problem 14 reduces to (3). Therefore (3) is a special case of (14). Moreover, if $K=K_{1}=1$, this problem further reduces to scalar (i.e., non-group) sparse denoising. In the following discussion, we consider the group sparse case: $N \gg K \geqslant K_{1}>0$. The case $K_{1}=0$ is trivial.

In the following section, we exploit the convexity condition of $(14)$, to constrain the non-convex penalty function $\phi$, so as to ensure that the objective function $P_{1}$ is convex. Therefore, the problem formulation we ultimately propose is a convex optimization problem. Hence, it will have no non-optimal local minima in which an iterative optimization algorithm can be trapped.

\subsection{Convexity conditions}

Proposition 2. Let $b \in\{0,1\}^{K}$ be a binary vector with $\sum_{k} b_{k}=K_{1}$. The function $P: \mathbb{R}^{K} \rightarrow \mathbb{R}$ defined as

$$
P(u)=\frac{1}{2 K_{1}} \sum_{k=0}^{K-1} b_{k} u_{k}^{2}+\lambda \phi\left(\left[\sum_{k=0}^{K-1} b_{k} u_{k}^{2}\right]^{1 / 2} ; a\right),
$$

is strictly convex if

$$
\phi^{\prime \prime}(x ; a)>-\frac{1}{K_{1} \lambda} \quad \text { for all } x \neq 0
$$


This Proposition is proved in detail in Appendix B. Utilizing the above results, we find a range of $a$ ensuring that, even though $\phi$ is non-convex, the objective function $P_{1}$ in 14 is strictly convex.

Theorem 1. Assume that problem $P_{1}$ (14) has $K_{1}=\sum_{k} b_{k}$ non-zero weights in one binary weighted group, then the objective function is strictly convex if the parameter ' $a$ ' of the penalty function $\phi(\cdot ; a)$ satisfies

$$
0 \leqslant a<\frac{1}{K_{1} \lambda}
$$

Proof. First, since $\sum_{k} b_{k}=K_{1}$ and $b_{k} \geqslant 0, x_{n}^{2} \geqslant 0$, it follows that

$$
K_{1} \sum_{n} x_{n}^{2}=\sum_{k} b_{k} \sum_{n} x_{n}^{2}=\sum_{n} \sum_{k} b_{k} x_{n}^{2} .
$$

Using 21], we write $\frac{1}{2} \sum_{n} x_{n}^{2}=\frac{1}{2 K_{1}} \sum_{n} \sum_{k} b_{k} x_{n}^{2}$. Therefore, the data fidelity term in problem (14) can be written as

$$
\begin{aligned}
F(x) & =\frac{1}{2}\|y-x\|_{2}^{2}=\frac{1}{2} \sum_{n} x_{n}^{2}+L(x) \\
& =\frac{1}{2 K_{1}} \sum_{n=0}^{N-K}\left(\sum_{k} b_{k} x_{n+k}^{2}\right)+L(x),
\end{aligned}
$$

where $L(x)$ is linear in $x$. Adding $L(x)$ to a strictly convex function yields a strictly convex function.

Using the above results, the objective function in problem (14) can be reorganized as

$$
\begin{aligned}
P_{1}(x) & =\sum_{n=0}^{N-K}\left[\frac{1}{2 K_{1}} \sum_{k} b_{k} x_{n+k}^{2}+\lambda \phi\left(\left[\sum_{k} b_{k} x_{n+k}^{2}\right]^{1 / 2} ; a\right)\right]+L(x), \\
& =\sum_{n=0}^{N-K} P\left(B_{n}(x)\right)+L(x),
\end{aligned}
$$

where $B_{n}(x)$ is defined in (16). As a consequence, if $P$ is strictly convex, then $P_{1}$ is strictly convex. The condition for convexity of $P$ is given in Proposition 2 . Hence, as long as the inequality condition (19) is satisfied, $P$ is strictly convex. Moreover, $\phi$ satisfies condition $\phi^{\prime \prime}(x ; a) \geqslant-a$ (condition 6 in Section 2). This implies that when 20 is satisfied, $P$ is strictly convex, and the entire objective function $P_{1}$ is convex.

Note that Theorem 1 generalizes the convexity condition of OGS in [10, Corollary 2]. When every element in binary vector $b$ equals 1 then $K_{1}=K$ and Theorem 1 reduces to Corollary 2 in 10 as a special case.

We have proved that under a more flexible group structure (binary weights), non-convex penalty functions can be utilized to promote structured sparsity, and the convexity of the objective function is preserved when the regularization parameter $a$ is suitably set. Moreover, the result also shows that when maximizing the non-convexity of the penalty function, only the the nonzero weights matter for the selection of the parameter $a$. 


\subsection{Algorithm derivation}

To minimize $P_{1}$ using the MM procedure, we define a majorizer $G: \mathbb{R}^{N} \times \mathbb{R}^{N} \rightarrow \mathbb{R}$, defined by

$$
G(x, v)=\frac{1}{2}\|y-x\|_{2}^{2}+\lambda \sum_{n} g(\theta(x, b, n), \theta(v, b, n)) .
$$

We can write $G$ as

$$
\begin{aligned}
G(x, v) & =\frac{1}{2}\|y-x\|_{2}^{2}+\frac{\lambda}{2} \sum_{n} \frac{1}{\psi(\theta(v, b, n))} \theta^{2}(x, b, n)+C \\
& =\frac{1}{2}\|y-x\|_{2}^{2}+\frac{\lambda}{2} \sum_{n} \sum_{k} \frac{b_{k}}{\psi(\theta(v, b, n))} x_{n+k}^{2}+C \\
& =\frac{1}{2}\|y-x\|_{2}^{2}+\frac{\lambda}{2} \sum_{n}[r(v)]_{n} x_{n}^{2}+C
\end{aligned}
$$

where $r(v) \in \mathbb{R}^{N}$ is defined as

$$
[r(v)]_{n}:=\sum_{j=0}^{K-1} \frac{b_{j}}{\psi(\theta(v, b, n-j))} .
$$

Then $G(x, v)$ can be written as

$$
\begin{aligned}
G(x, v) & =\frac{1}{2} \sum_{n} x_{n}^{2}+\frac{\lambda}{2} \sum_{n} r_{n} x_{n}^{2}-\sum_{n} y_{n} x_{n}+C(y) \\
& =\sum_{n}\left(\frac{1}{2}+\frac{\lambda[r(v)]_{n}}{2}\right) x_{n}^{2}-y_{n} x_{n}+C(y)
\end{aligned}
$$

which has an explicit minimizer $x_{n}=y_{n} /\left(1+\lambda[r(v)]_{n}\right)$. Hence, the MM iteration (5) is given by

$$
x_{n}^{(i+1)}=\frac{y_{n}}{1+\lambda\left[r\left(x_{n}^{(i)}\right)\right]_{n}} .
$$

Table 2 gives the explicit steps to solve (14), assuming the penalty function is chosen from Table 1 and satisfies (20). This guarantees the problem (14) is strictly convex and consequently MM procedure (5) will converge to the unique global minimizer.

Note that zero-locking might occur when using quadratic function to majorize non-smooth function [19]. For the OGS problem, initializing the algorithm by $x^{(0)}=y$ avoids this issue; a detailed proof is given in [10, Lemma B]. This lemma is not affected by introducing binary group weights. In other words, if the function $F$ in [10, Lemma B] is substituted with $P_{1}$ (14), the derivation is still valid with an almost identical proof. Moreover, since we allow $\psi(x)$ to be 0, Equation (26) may lead to a 'divide-by-zero'. The work of 10 contains a sufficient discussion that this problem is avoided by the initialization $x^{(0)}=y$, based on a same lemma. Hence, there is no zero-locking or 'divide-by-zero' issue when solving the problem (14) by the algorithm in Table 2

\subsection{Periodicity-induced OGS (POGS)}

In the previous sections, we have given a method for group sparse denoising with binary weights within the group. In signal model (11), since the periodicity of impulsive faults in $x$ is assumed to be approximately 
Table 2: OGS with binary weights.

Input: $y \in \mathbb{R}^{N}, \lambda, b \in\{0,1\}^{K}$
Initialization: $x=y, S=\left\{n: y_{n} \neq 0\right\}$
Repeat for $n \in S:$
$\quad r_{n}=\sum_{j=0}^{K-1} \frac{b_{j}}{\psi(\theta(x, b, n-j))}$
\[ x_{n}=\frac{y_{n}}{1+\lambda r_{n}} \]
$\quad S=\left\{n:\left|x_{n}\right|>\epsilon\right\}$
Until convergence
Return: $x$

consistent over a reasonable duration, the time interval between two consecutive faults can be considered identical within the support of a group. Moreover, when the period $T$ of a potential fault is known or predictable from the knowledge of the machinery, we can select the group with a length $K$ and its zero and non-zero entries by

$$
\begin{aligned}
& N_{0}+N_{1} \approx f_{s} T, \\
& N_{0}+N_{1}=K / M,
\end{aligned}
$$

where $N_{1}$ and $N_{0}$ are the estimated length (in samples) of impulsive transients and the time interval between them, and integer $M \geqslant 2$ is the number of periods contained in one group, and $f_{s}$ is sampling rate. Thus, in one group, the numbers of zero and non-zero entries are $K_{0}=M N_{0}$ and $K_{1}=M N_{1}$, respectively.

Moreover, when the transient sequence is periodical, the binary weight $b_{k}$ are chosen according to a periodic group structure. Specifically, under the constraint $31, b \in\{0,1\}^{K}$ is given by

$$
b=[\underbrace{1,1, \ldots, 1}_{N_{1}}, \underbrace{0,0, \ldots, 0}_{N_{0}}, \underbrace{1,1, \ldots, 1}_{N_{1}}, \underbrace{0,0, \ldots, 0}_{N_{0}}, \cdots, \underbrace{1,1, \ldots, 1}_{N_{1}}, \underbrace{0,0, \ldots, 0}_{N_{0}}],
$$

where in each period, there are $N_{1}$ non-zero entries grouped according to the impulsive signal, and the entire group comprises $M$ periods. In this case, the last $N_{0}$ zeros in 32 has no effect in problem (14) by the definition (15). In practice, we omit trailing zeroes and the actual length of $b$ involved in computation is $K-N_{0}$. Note that although we use the parameters such as $K, K_{0}, K_{1}, N_{0}, N_{1}$ and $M$ to illustrate the binary pattern structure, in fact given $f_{s}$ and $T$, we only need to select $N_{1}$ and $M$, then use (31), the pattern of $b$ 32 can be determined.

Consequently, we propose to recover a periodic impulsive signature from a noisy observation by solving problem (14) with $b$ defined by (31) and (32). We refer to this method as Periodicity-induced OGS (POGS). This is an extension of OGS accounting for the periodicity of the sparse signal.

As a special case, if the period $T$ is not known, we may use conventional OGS (3) to detect faults. If a period $T$ can be determined by inspecting the output produced by OGS, then an enhanced result with better accuracy may be achieved by POGS using the determined periodicity. 

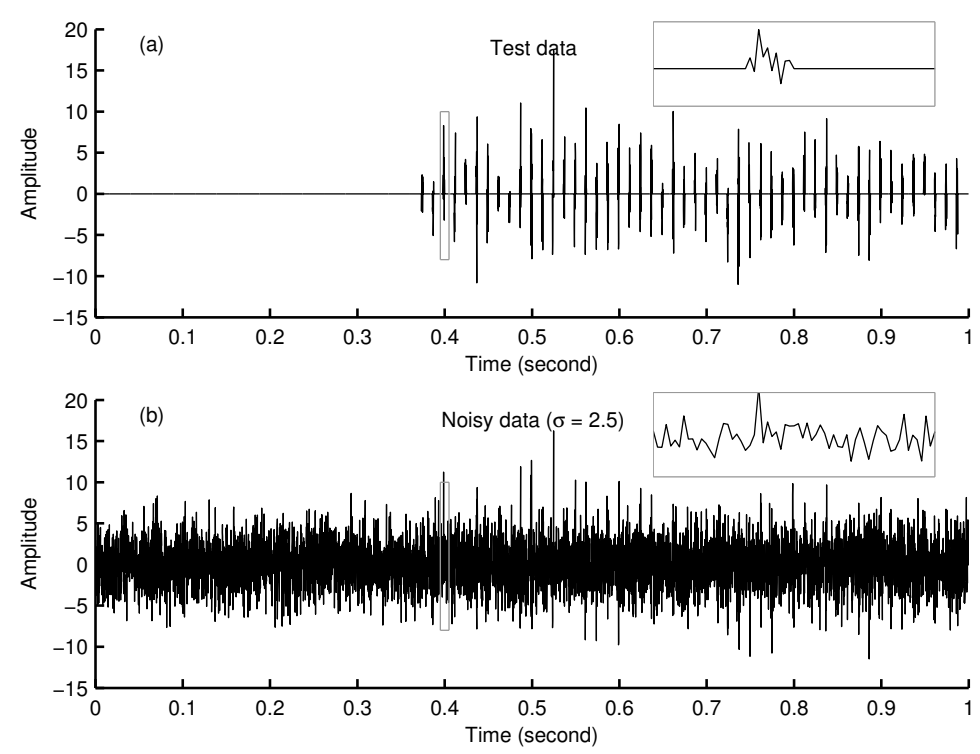

Figure 2: Example 1: Simulated signal: (a) clean data and (b) noisy data.

\section{Simulation validation}

To test the proposed method, we apply it to the simulated data illustrated in Fig. 2(a). The signal is a 1-second signal with sampling rate $f_{s}=6400 \mathrm{~Hz}$, and is composed of a periodic sequence of transients occurring with $80 \mathrm{~Hz}$.

We simulate the vibration signal containing features caused by machinery defect as a sequence of impulsive transients, and each transient consists for 10 samples $\left(1.6 \mathrm{~ms}\right.$ when $\left.f_{s}=6400 \mathrm{~Hz}\right)$. In this example, each transient is composed of a random number of sinusoidal components, each with random frequency and random initial phase. More specifically, each transient can be written as

$$
v(n)=\sum_{i=1}^{U} A_{i} \sin \left(\omega_{i} n+\beta_{i}\right), \quad n=0,1,2 \ldots 9,
$$

where $1 \leqslant U \leqslant 10$ is a random integer, and for each $i, A_{i}$ is a random amplitude, and $\omega_{i}$ is a random frequency, and $\beta_{i}$ is a random initial phase. The sequence of transients is shown in Fig. 2(a), and we show the detail of one transient at about $t=0.40$ second in the box. The generated test signal is multiplied by a constant, so that it has unit standard deviation.

In order to evaluate the false alarm rate, the first part of the test signal contains no transient. Fig. 2(a) shows the clean test signal, where there are 50 faults starting at approximately $t=0.36$ second with a period $T=1 / 80$ second. White Gaussian noise with standard deviation $\sigma=2.5$ is added to the simulated fault sequence, as illustrated in Fig. 2(b).

When the periodicity of the faults is known, we can use POGS, where (31) and 32 are used to define the binary weight vector $b$. In this example, we set $N_{1}=4$ and $M=4$. Since $T=1 / 80$ seconds, we can calculate $N_{0}=6400 / 80-N_{1}=80-4=76$ according to conditions (31), then the explicit pattern of $b$ can be determined as Fig. 4. The denoising result is shown in Fig. 3(a), and the transients can be easily identified with an almost pure zero baseline. In this example, we use OGS and POGS with the atan penalty function and non-convexity parameter $a$ set to its maximum value of $1 /\left(K_{1} \lambda\right)$, so as to maximally induce sparsity subject to the constraint that the objective function $P_{1}$ is convex. 

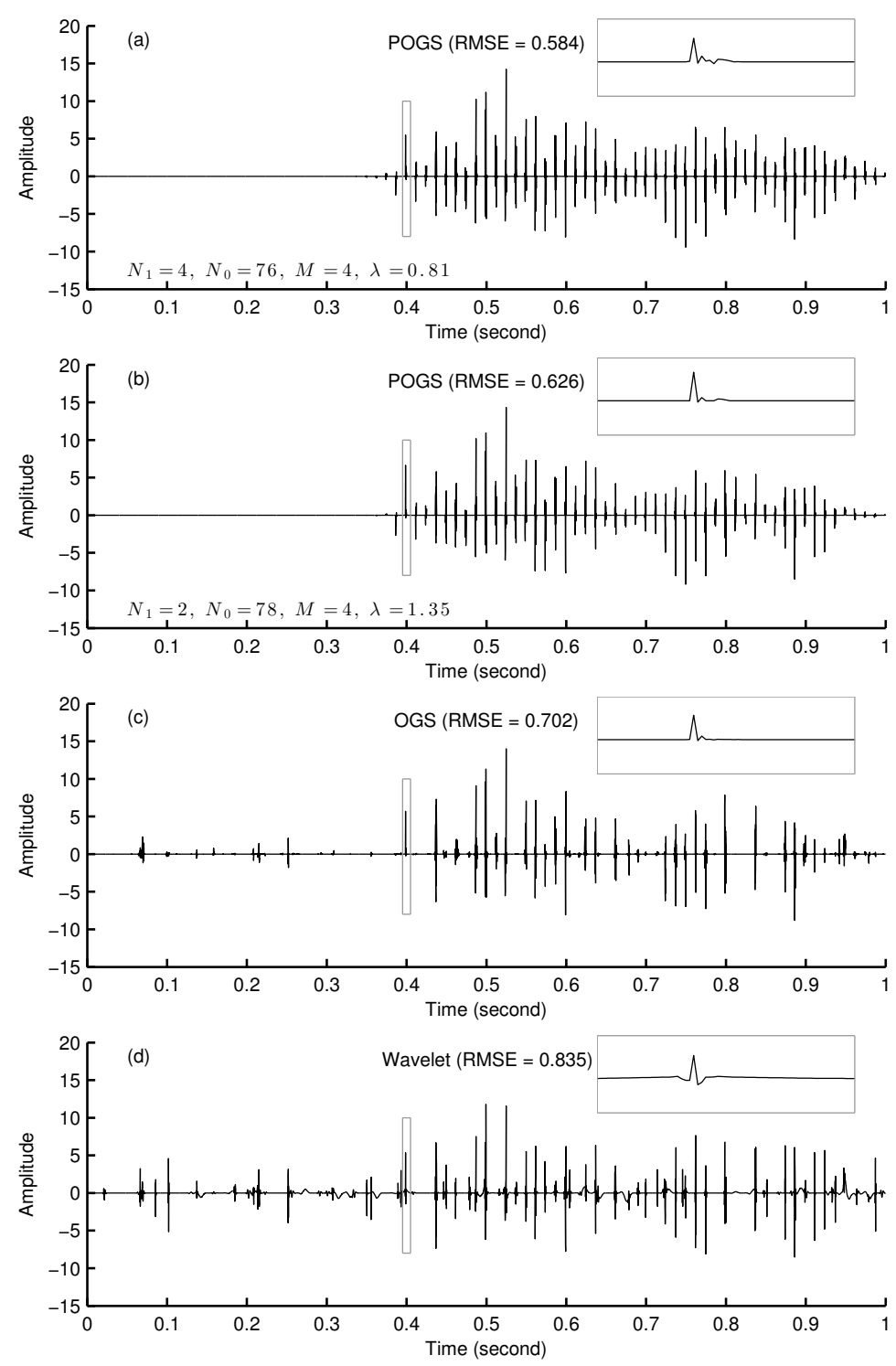

Figure 3: Example 1: Denoising results. (a) Proposed method with $N_{1}=4, M=4$. (b) Proposed method with $N_{1}=2, M=4$. (c) Conventional OGS. (d) Wavelet-based denoising.

Fig. 3(b) shows another example using $N_{1}=2$ to determine the pattern $b$. The result is slightly worse in RMSE than Fig. 3(a), because $N_{1}=2$ in this example does not match the simulated data. However, $N_{1}=2$ is the lower limit of a realistic value, because in practice it is very rare that the transients are all single-sample spikes, when the data is properly measured. As a consequence, the result in Fig. 3. (b) can be understand as the worst case of choosing an inappropriate $b$.

Fig. 3(c) shows the result of denoising using conventional non-periodic OGS (3) with group size $K=8$ and the arctangent (atan) non-convex penalty function. The regularization parameter $\lambda$ is chosen by the look-up table in [9, which sets $\lambda$ proportional to the noise $\sigma$. In this example, we set $\lambda=0.52 \sigma$. The result in Fig. 3(c) misses some faults and yields several false detections, e.g., the ones at about 0.82 second, and some false transients appear before $t=0.36$ second.

As a comparison, we adopt conventional wavelet-based denoising method to the test signal. More specif- 


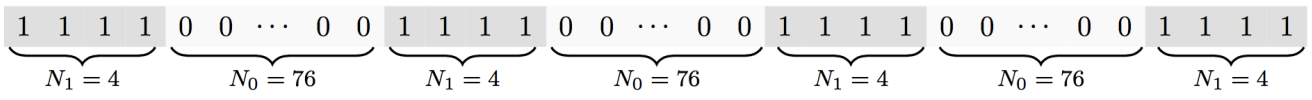

Figure 4: Example 1: Binary pattern $b$ when $M=4, N_{1}=4$.
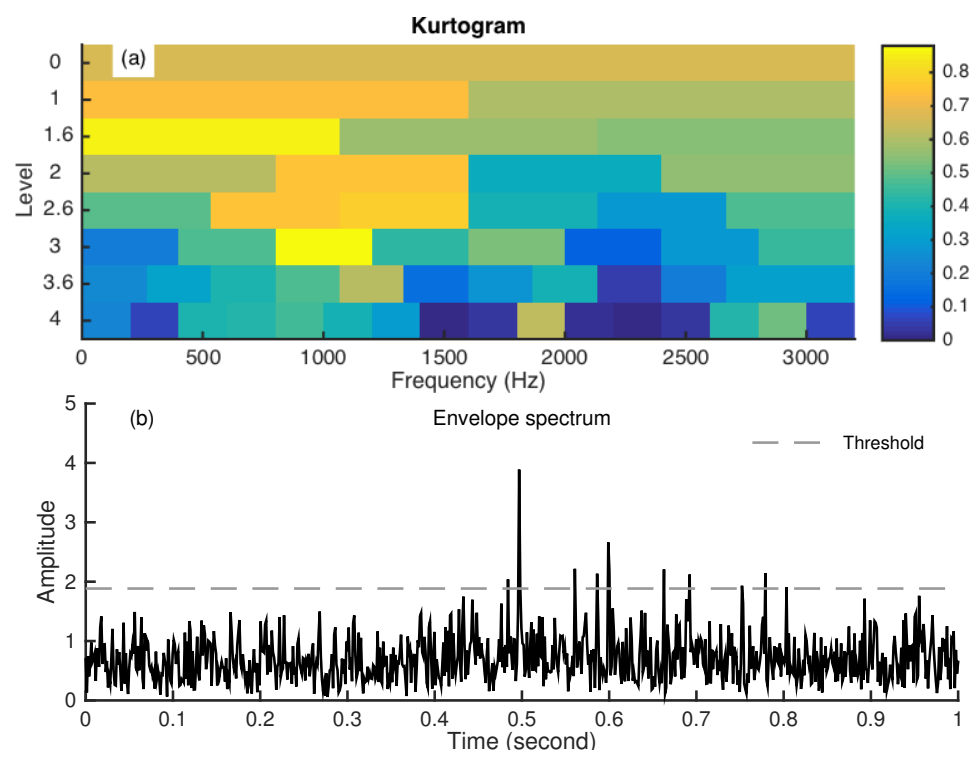

Figure 5: Example 1: Results of fast spectral kurtosis. (a) Kurtogram. (b) Envelope of extracted transients.

ically, a 5-scale undecimated wavelet transform 20] with 3 vanishing moments is used, and the result is shown in Fig. 3(d). For denoising, we apply hard-thresholding and chose the threshold value by $3 \sigma$-rule for each subband. In Fig. 3(d), although some large amplitude transients can be recovered at correct locations, they exhibit the same shape as the chosen wavelet [see the box in Fig. 3(d)].

We compare the performance with fast spectral kurtosis [21] This method produces the kurtogram in Fig. 5(a), where the kurtosis maximum is at the third level with an estimated 'optimal carrier frequency' at $1000 \mathrm{~Hz}$ [see the bright yellow area in Fig. 5(a)]. The corresponding amplitude of the extracted transients is shown in Fig. 5(b), with an automatically generated threshold shown as a gray dashed line. The peaks after $t=0.36$ seconds have a greater density, which indicates that it is more likely that faults occur after $t=0.36$ seconds. However, the useful repetitive transients are surrounded by strong irrelevant noise.

\subsection{Parameter selection}

Setting pattern $b$. In many cases of fault diagnosis, it is feasible to estimate the period of the transients based by component geometry and rolling speed, but it might be difficult to estimate the duration of the transient. However, since the binary structure $b$ is acting as a sliding window capturing the global periodicity structure, it is not necessary to match the length of a transient exactly. As a consequence, we suggest to constrain the value of $N_{1}$ relatively small, e.g. $2 \leqslant N_{1} \leqslant 4$. However, if the sampling rate is quite high and a transient may contain more samples, then the value of $N_{1}$ can be specified greater than 4 . The value of $M$ determines the number of non-zero entries (the value of $K_{1}$ ) in $b$, and $K_{1}$ effects the non-convexity of the regularizer in the proposed problem (14). In our experiments, we keep use $M=4$.

Setting regularization parameter $\lambda$. In order to explore the correlation of the regularization param- 

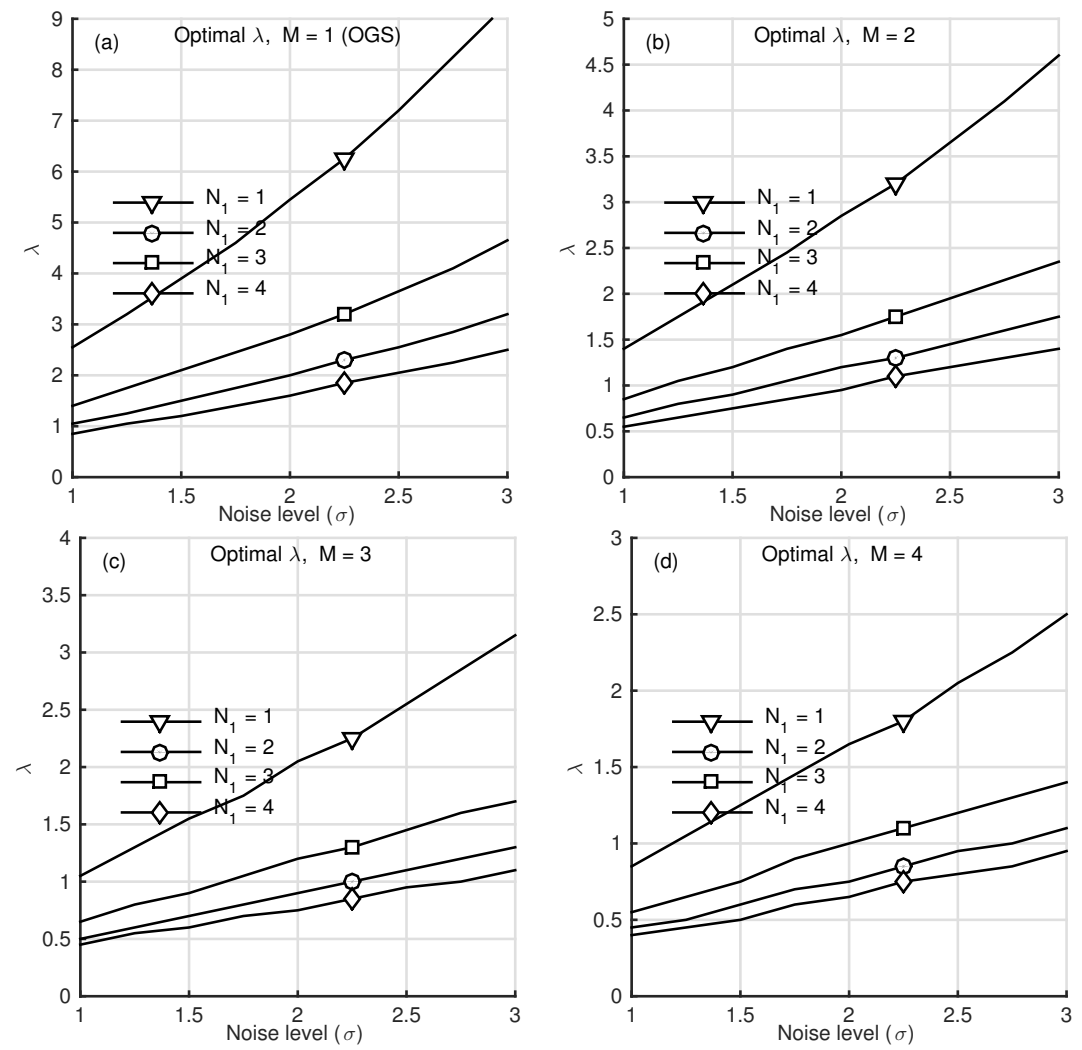

Figure 6: Example 1: Optimal $\lambda$ at different noise level. (Note that the vertical axes of the sub-figures are different.)

eter $\lambda$ to the binary pattern $b(32)$, we show the optimal $\lambda$ as a function of the standard deviation $\sigma$ of noise using different binary patterns in Fig. 6. We define the optimal $\lambda$ as the value minimizing the RMSE for each fixed binary pattern $b$.

In this test, using the data in Fig. 2(a), we search for $\lambda$ minimizing an average RMSE (20 trials) at each noise level generated by different random seeds. We present the results of select $M$ from 1 to 4 in Fig. 6(a) to (d), respectively. In each figure, we show the optimal $\lambda$ as a function of $\sigma$ under different $N_{1}$. Note that, in Fig. 6(a), when $M=1$ and $N_{1}=1$, the proposed method adheres neither grouping nor periodic structure, in which case, the problem (14) coincides to the BPD problem with non-convex regularizer.

Note that since we simulate the test signal with a unit standard deviation, the horizontal axis in Fig. 6 is also the ratio of deviation from noise to data. In other word, we can use Fig. 6 as a look-up table, when the input noise-to-signal ratio (SNR) is known.

Moreover, all the 16 curves in Fig. 6 show that the optimal $\lambda$ varies approximately linearly with noise level. In practice, we suggest to chose $\lambda$ in $(14)$ proportional to the noise level as $\lambda=r \sigma$. Through further experiments, we provide Table 3 as a guide for choosing the multiplier $r$.

\subsection{Receiver operating characteristic (ROC) evaluation}

The above comparative evaluation of OGS, POGS, wavelet, and fast spectral kurtosis uses RMSE as an indicator of denoising performance. However, the RMSE by itself is not a sufficient indicator of fault detection accuracy. In the following, we use a receiver operating characteristic (ROC) based approach to evaluate the relative accuracy of the methods. 
Table 3: Selection of $r$ for setting $\lambda$.

\begin{tabular}{cccccc}
\hline & $N_{1}$ & 1 & 2 & 3 & 4 \\
\hline 1 & & & & & \\
\hline 1 & 3.700 & 1.700 & 1.150 & 0.925 \\
2 & & 1.700 & 0.850 & 0.625 & 0.475 \\
3 & & 1.150 & 0.625 & 0.450 & 0.375 \\
4 & & 0.925 & 0.475 & 0.375 & 0.325 \\
\hline
\end{tabular}

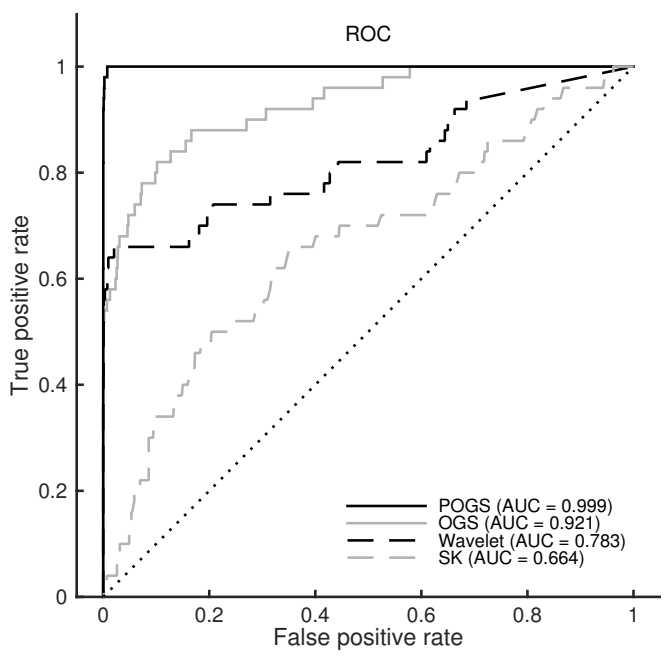

Figure 7: Example 1: ROC curves of faults detection.

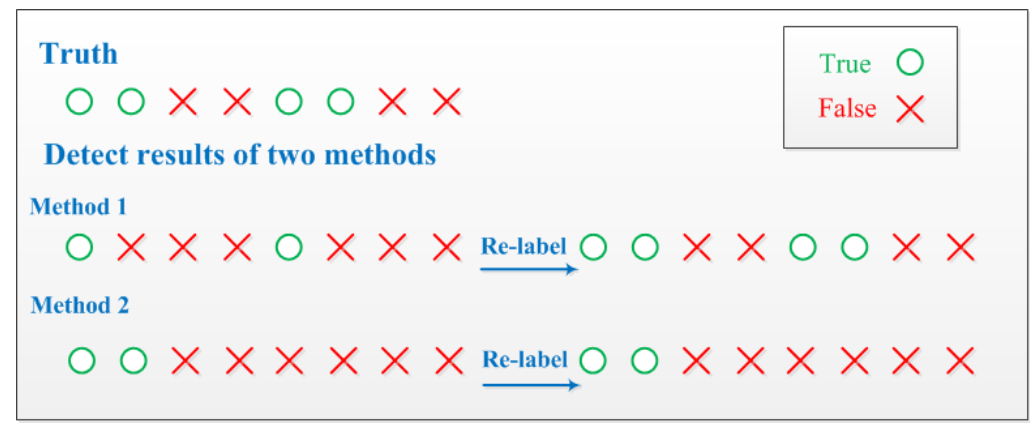

Figure 8: Example of classification rule.

The receiver operating characteristic (ROC) curve is a well known detection performance evaluation methodology 22]. ROC curves are well suited to the problem of vibration-based diagnosis applications 23]. An ROC curve is generated by plotting the probability of a false alarm against the probability of detection as the threshold level is varied. Since the POGS approach is focused on machinery fault detection, the ROC curve is utilized to validate the superior detection performance of POGS compared to other methods. We define the classification rule as: if one sample in a transient [generated by [33] ] is detected as positive, then this entire transient a fault feature is detected and all the remaining samples are all assigned to be positive.

This rule is slightly different to the one used in [23, since using the sample-wise decision rule in 23] may cause problem of overweighting sample recovery, but neglecting detecting fault features as transients of a 
Table 4: The parameters of the tested bearing

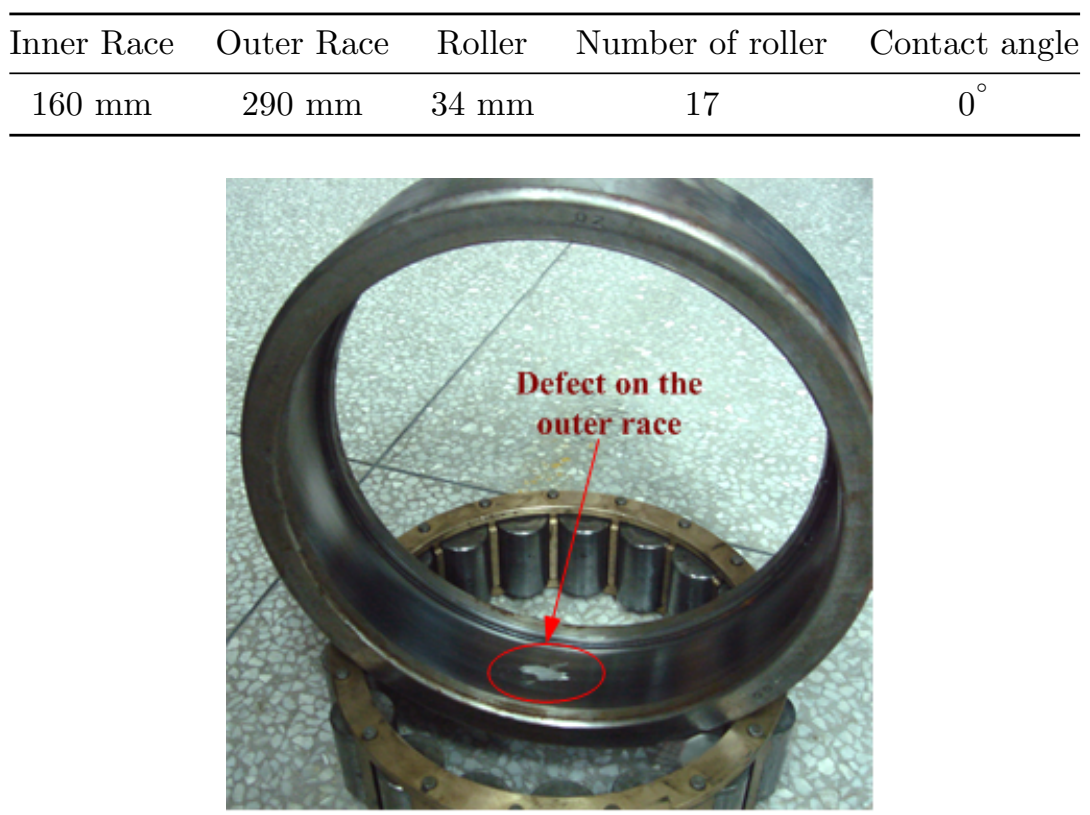

Figure 9: Example 2: Fault on the outer race of the testing bearing.

cluster of samples. We use a simple example in Fig. 8 to illustrate the problem and our classification rule. Suppose that the true signal has 8 samples consists 2 periods ( 4 samples for each period), and each period has 2 samples positive (labeled as circles). If Method 1 detects 2 samples belonging to two different transients respectively, and method 2 detects 2 samples belonging to only one transients, then the rule of [23] will give that the two methods have identical accuracy, because if merely counting the samples, they have a same number of samples detected. This is undesirable, since Method 2 misses an entire transient. To overcome this issue, we re-label the detect result, where if any sample in a transient of each period is detected, we re-label all the samples in the entire transient to be detected. In the example of Fig. 8 , after the re-labeling, Method 1 has a better accuracy because it detects both of the transients.

Fig. 7 shows the ROC curves from using OGS, POGS and wavelet-based method respectively. POGS achieves an almost perfect detection result. Also, OGS is better than wavelet-based method. In this example, because the results from POGS with different parameter settings [in Fig. 33(a) and Fig. 3(b)] obtain almost identical ROC curves, we chose to show the one in Fig. 3(a) where $N_{1}=4$ and $M=4$.

We also use the extracted envelope from fast spectral kurtosis method to perform a similar ROC analysis. Note that although we plot it together with other ROC curves in Fig. 7, since its envelope has a different length than the other results, the ROC curve is generated by a different number of samples.

\section{Experimental and engineering data validation}

\subsection{Example 2: Rolling bearing with defect on outer race}

In this example, the proposed approach is applied to a vibration signal collected from a locomotive rolling element bearing with defect. The testing locomotive rolling bearing with a slight scrape on its outer race is shown in Fig. 9. The vibration signal is measured from acceleration sensors, using SONY Ex data acquisition 

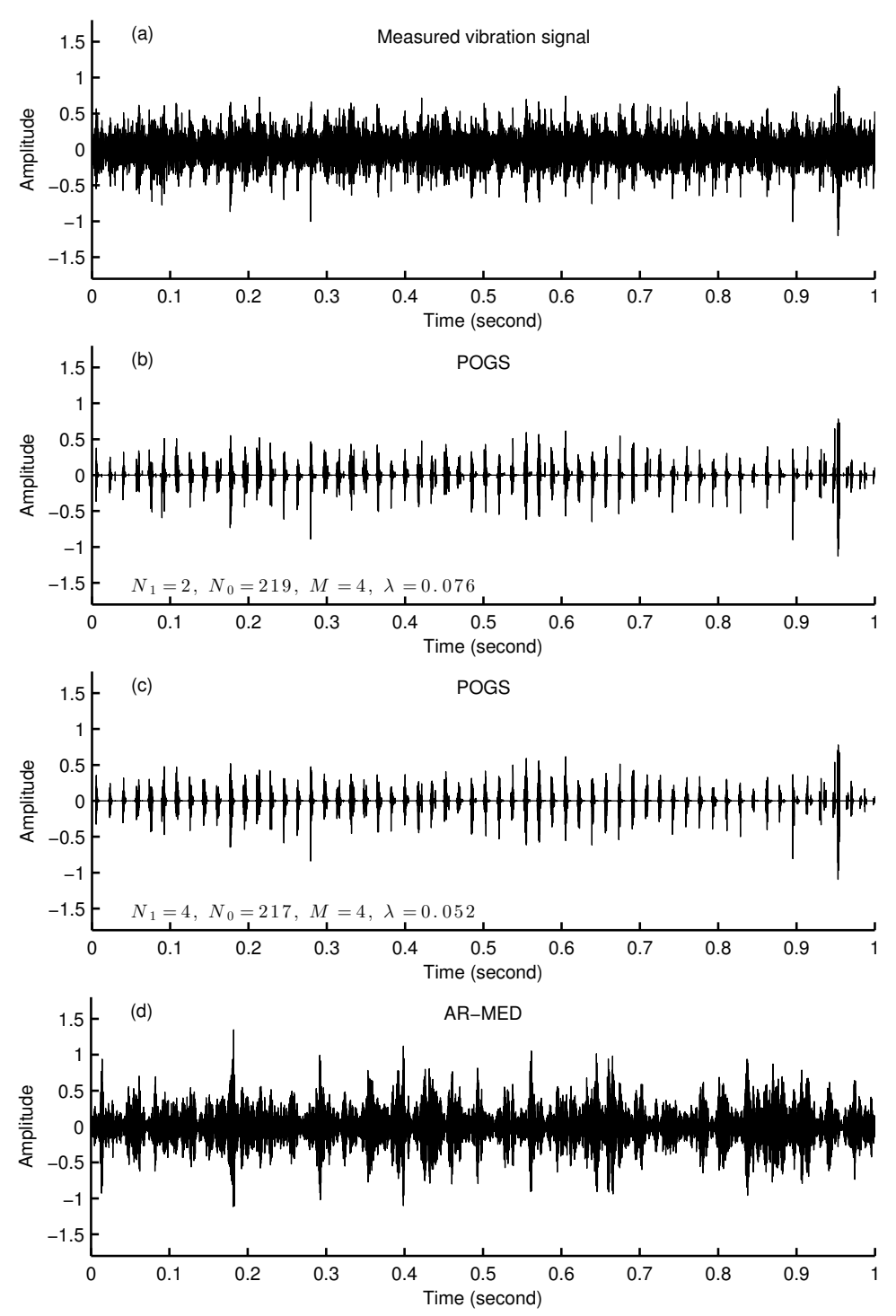

Figure 10: Example 2: (a) Test data. (b) Result of proposed method with $N_{1}=2, M=4$. (c) Result of proposed method with $N_{1}=4, M=4$. (d) Result of AR-MED.

system when the electric locomotive was running. The bearing type is $552732 \mathrm{QT}$ and its specification is shown in Table 4. The sampling rate is $12.8 \mathrm{kHz}$ and the current rotating speed is approximately $481 \mathrm{rpm}$. Thus, based on the geometric parameters and rotational speed, the characteristic frequency of the outer race defect is calculated to be $f_{o}=57.8 \mathrm{~Hz}$.

To adopt the proposed POGS (14), if the duration of a transient is uncertain, we can directly chose the pattern $b \sqrt{32}$ with $M=4$ and $N_{1}=2$, then by Table 3 we can chose $\lambda$ with the respect to the noise level. Note that since the 'noise' in the vibration data for fault detection is the background out of the transient sequence, in practice it can be easily estimated using healthy data.

In this example, we illustrate how to determine $\lambda$ without healthy data. We estimate the deviation of background by the formula

$$
\hat{\sigma}=\operatorname{MAD}(y) / 0.6745
$$




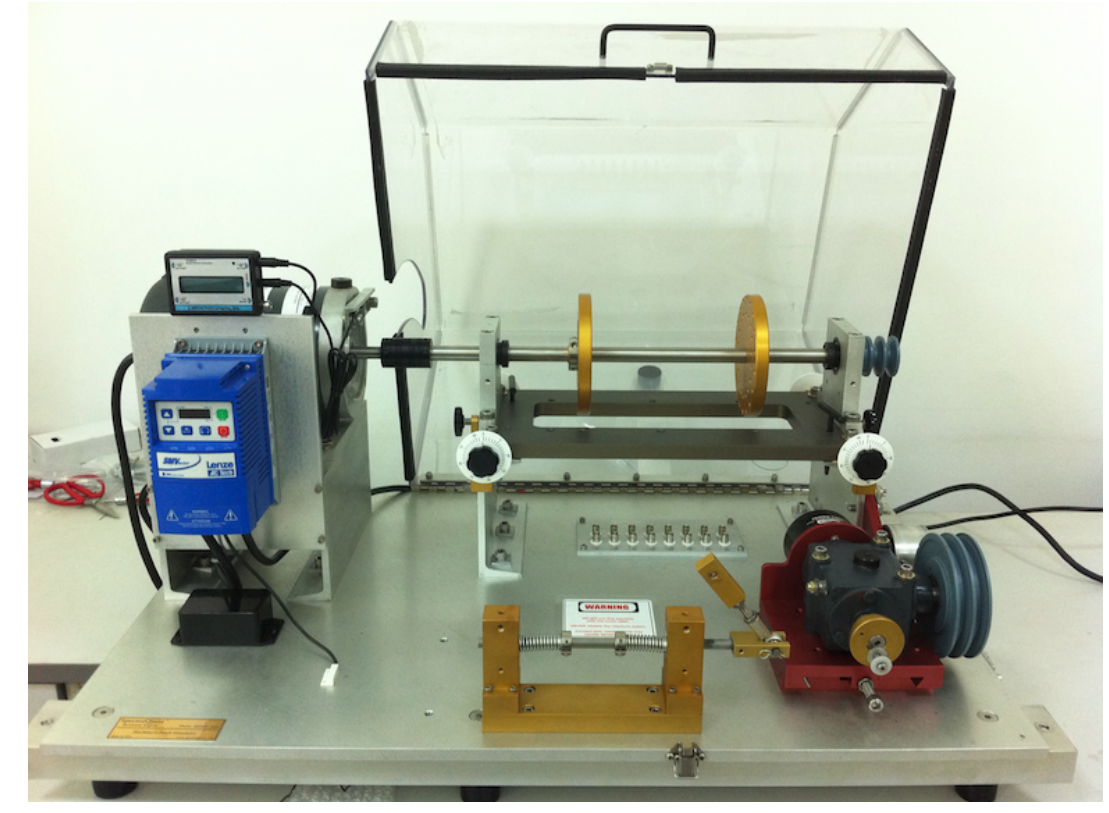

Figure 11: Example 3: Spectra Quest's machinery fault simulator.

which is a conventional estimator of noise level used for wavelet-based denoising 24, where MAD in (34) stands for median absolute deviation, defined as

$$
\operatorname{MAD}(y):=\operatorname{median}\left(\left|y_{n}-\operatorname{median}(y)\right|\right)
$$

In this example, the estimated deviation of background is $\hat{\sigma}=0.1606$ obtained by (34). The parameter $\lambda$ can be obtained using $\lambda=r \hat{\sigma}$ where $r=0.475$ (from Table 3). The result is shown in Fig. 10.b), where a periodical phenomenon can be easily identified. Moreover, we also test POGS with $M=4$ and $N_{1}=4$, using the same method to set $\lambda$. The result is shown in Fig. 10.c).

As a comparison, we also test the data with autoregression model assisted minimum entropy deconvolution (AR-MED) 251 The order of AR filter is chosen by maximizing Kurtosis value as the implementation suggested, and the estimated filter in the MED step has a length of 50. The result is shown in Fig. 10(d), where most of the impulses are promoted after deconvolution. However, since the baseline is still relatively noisy, the regularity of periodicity is not very clear.

\subsection{Example 3: Motor bearing with multiple faults}

Table 5: Fault frequencies for MFS motor bearing

\begin{tabular}{lcccc}
\hline Component & FTF & BPFO & BPFI & BSF \\
\hline Motor Bearings & 0.384 & 3.066 & 4.932 & 2.03 \\
\hline
\end{tabular}

To further demonstrate the effectiveness of the proposed method for machinery fault detection, applications of motor bearing fault diagnosis are studied in this section. The experiment is performed on a

\footnotetext{
${ }^{1}$ Implementation available online http://www.mathworks.com/matlabcentral/fileexchange/41614-ar-filter-+ -minimum-entropy-deconvolution-for-bearing-fault-diagnosis
} 

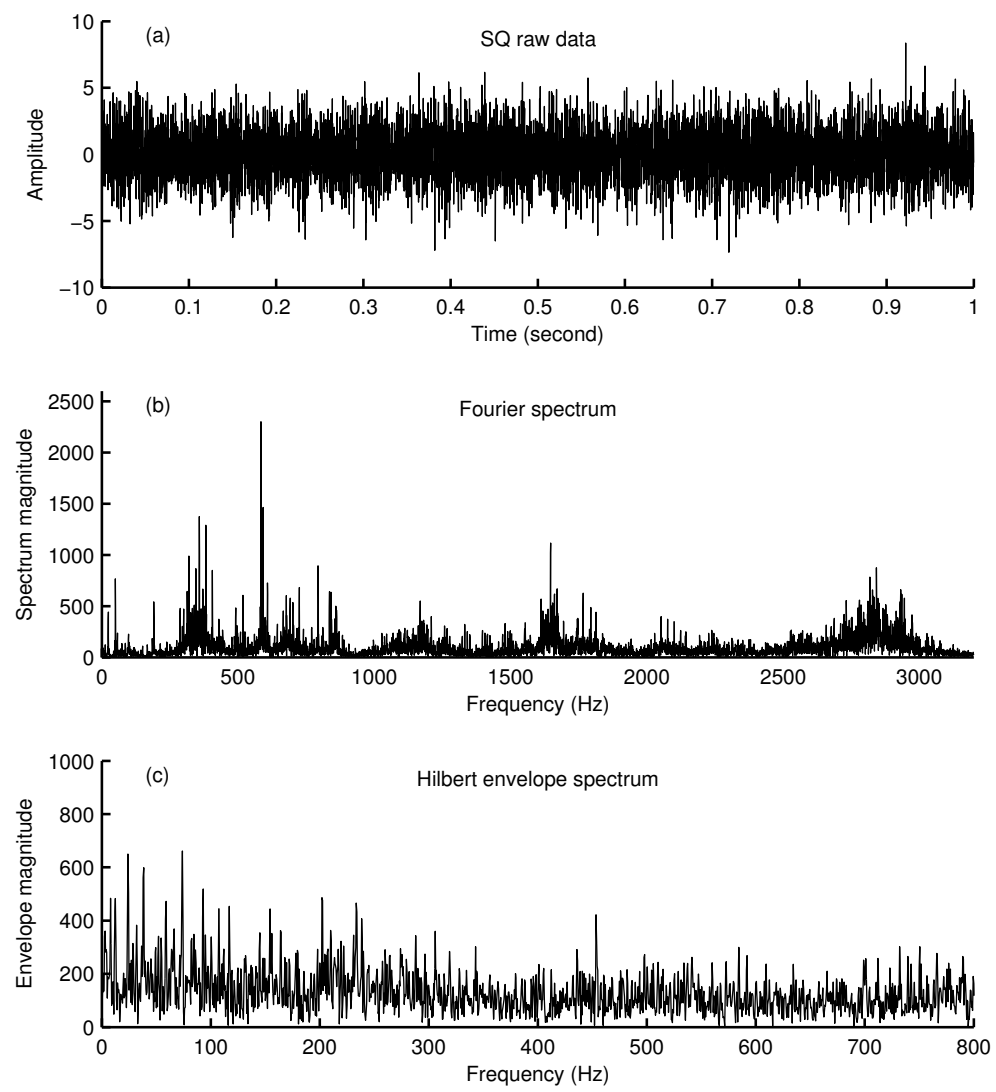

Figure 12: Example 3: Test data. (a) The measured vibration signal of the motor housing. (b) Fourier spectrum. (c) Hilbert envelope spectrum.

Spectra Quest's machinery fault simulator (MFS) illustrated in Fig. 11. The user does not need to make any modifications to the motor provided. The simulation setup consists of a motor with intentionally faulted bearings: one bearing with an inner race fault, and one bearing with an outer race fault. Therefore, the fault diagnosis of the motor bearings is equivalent to a compound faults detection case. The motor bearing fault frequencies for MFS components are given in Table 5 Accelerometers were mounted on the motor housing. The vibration signals were measured at a sampling rate $f_{s}=6400 \mathrm{~Hz}$. The rotating speed of the motor is $1433 \mathrm{rpm}(23.89 \mathrm{~Hz})$. Hence, the characteristic frequencies of the inner race and outer race of the motor bearings are calculated to be $f_{i} \approx 117.8 \mathrm{~Hz}$ and $f_{o} \approx 73.2 \mathrm{~Hz}$, respectively.

An observed vibration signal with a duration of 1 second is illustrated in Fig. 12(a). However, the useful periodic pulses are buried in strong background noise and irrelevant interference. The frequency spectrum and the Hilbert envelope spectrum of the signal are shown in Fig. 12(b) and (c), respectively. It can be observed from Fig. 12(b) that the energy of the spectrum is distributed along the whole frequency range. Peaks at $24 \mathrm{~Hz}$ and its harmonics can be observed in the low frequency band, which correspond to the rotating frequency and its harmonics. The useful characteristic frequencies used to monitor the health status of motor bearings can not be observed in Fig. 12 (c).

The proposed POGS approach is utilized to process the vibration signal. The results are shown in Fig. 13 and Fig. 14. We run POGS twice with two group structures, determined by the inner race period $T_{i}$ and the outer race period $T_{o}$, for the purpose of separating the useful impulsive fault features. In particular, keeping $N_{1}=2$ and $M=4$, we define two different group structures using (31) and (32) based on the inner race 

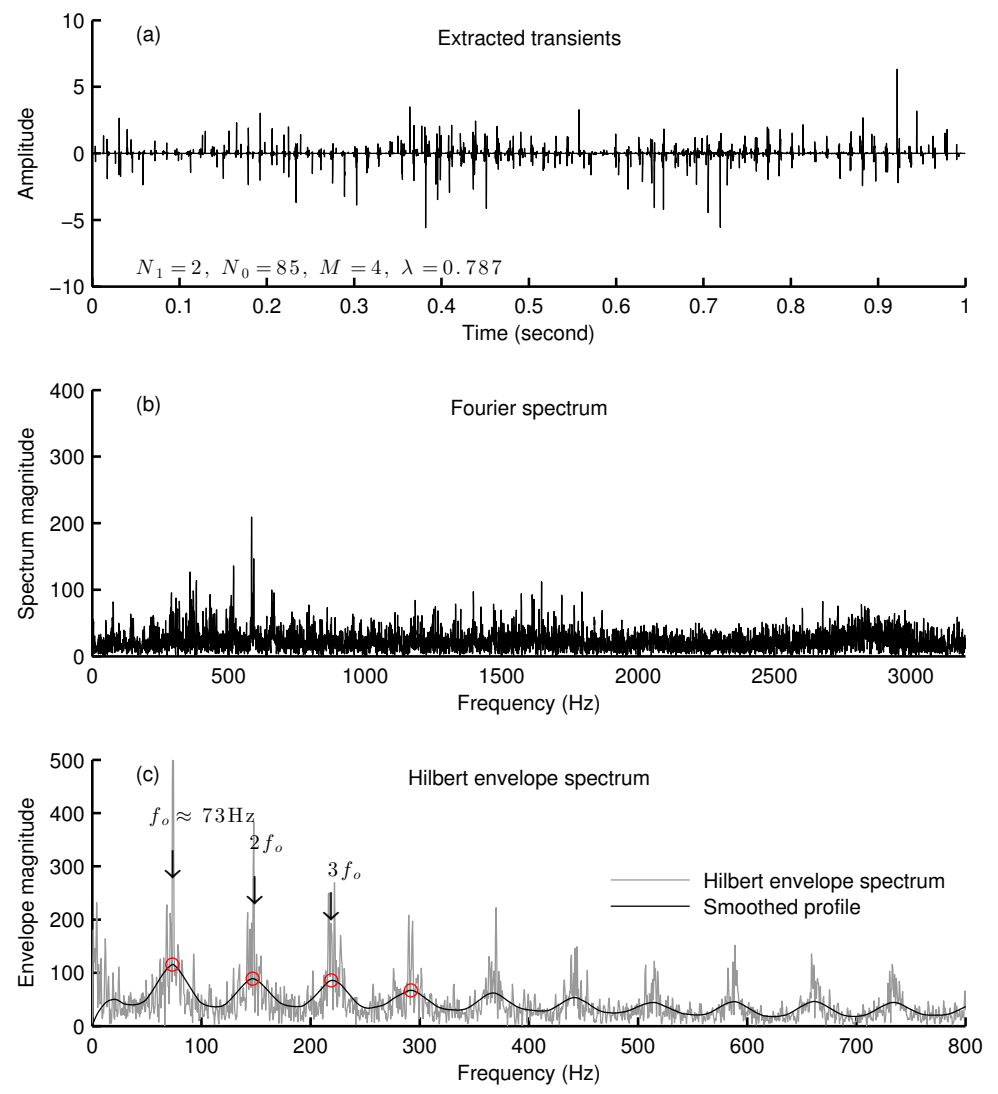

Figure 13: Example 3: Outer race faults detection $\left(N_{1}=2, M=4\right)$ : (a) Extracted transients, (b) Fourier spectrum and (c) Hilbert envelope spectrum.

period $T_{i}=1 / f_{i}$ and the outer race period $T_{o}=1 / f_{o}$, respectively. We use the atan penalty function with $a=1 /\left(K_{1} \lambda\right)$ to ensure convexity of the objective function. The value of $\lambda$ obtained using healthy data, is set so as to diminish the healthy data to almost all zeros.

The two periodic-related values correspond to the outer race defect and the inner race defect frequencies respectively. Strong periodic impulses with intervals of approximately 0.0133 second $(75 \mathrm{~Hz})$ are clearly revealed Fig. 13(a), which is exactly in accordance with the outer race characteristic frequency of $73.2 \mathrm{~Hz}$. Similarly, periodic transient features with the period 0.0085 second $(118 \mathrm{~Hz})$ can be observed in Fig. 14(a), which is approximately the inner race characteristic frequency of $117.8 \mathrm{~Hz}$. To further reveal the characteristic frequencies, the frequency spectrum and the Hilbert envelope spectrum of the processed signals are shown in Fig. 13.b) and (c) and Fig. 14(b) and (c). We also present the smoothed profiles of the Hilbert envelop spectrum to indicate the characteristic frequencies more clearly. The Hilbert spectrum of the processed result illustrated in Fig. 13.(c) is obtained using the proposed approach with prior knowledge of the outer race characteristic frequency $73.2 \mathrm{~Hz}$. Apparently, the characteristic frequencies of outer race $73 \mathrm{~Hz}$ and its harmonic components are clearly revealed, as shown in Fig. 13(c). Similarly, Fig. 14(c) is obtained utilizing the proposed approach with prior knowledge of the outer race characteristic frequency $117.8 \mathrm{~Hz}$. The characteristic frequencies of inner race $117 \mathrm{~Hz}$ and its harmonic components can be clearly observed in Fig. 14(c). Thus, the proposed periodic non-convex regularized OGS approach successfully detects the compound faults of the motor bearings. More specifically, the fault features of outer race defect and inner race defect are clearly separated utilizing the proposed approach. 

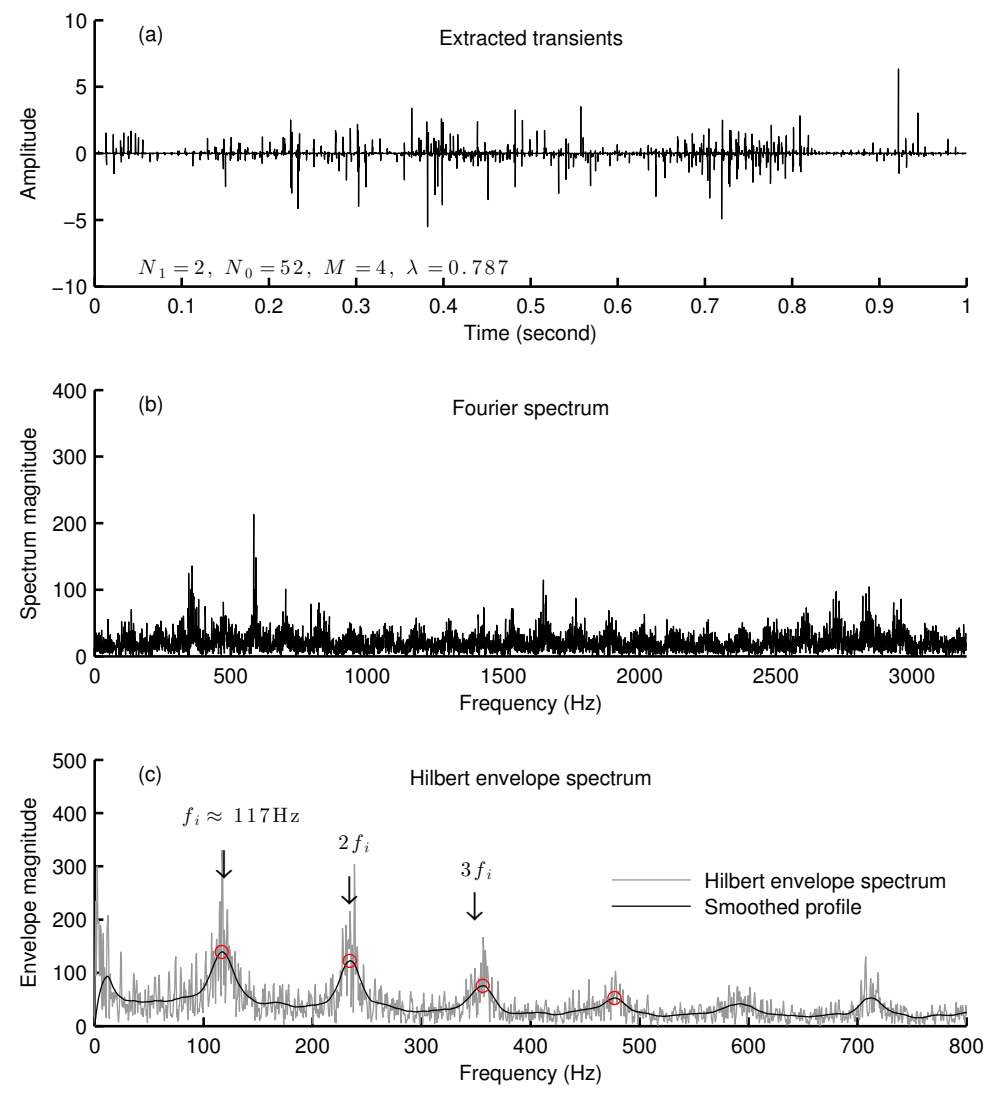

Figure 14: Example 3: Inner race faults detection $\left(N_{1}=2, M=4\right)$ : (a) Extracted transients, (b) Fourier spectrum and (c) Hilbert envelope spectrum.
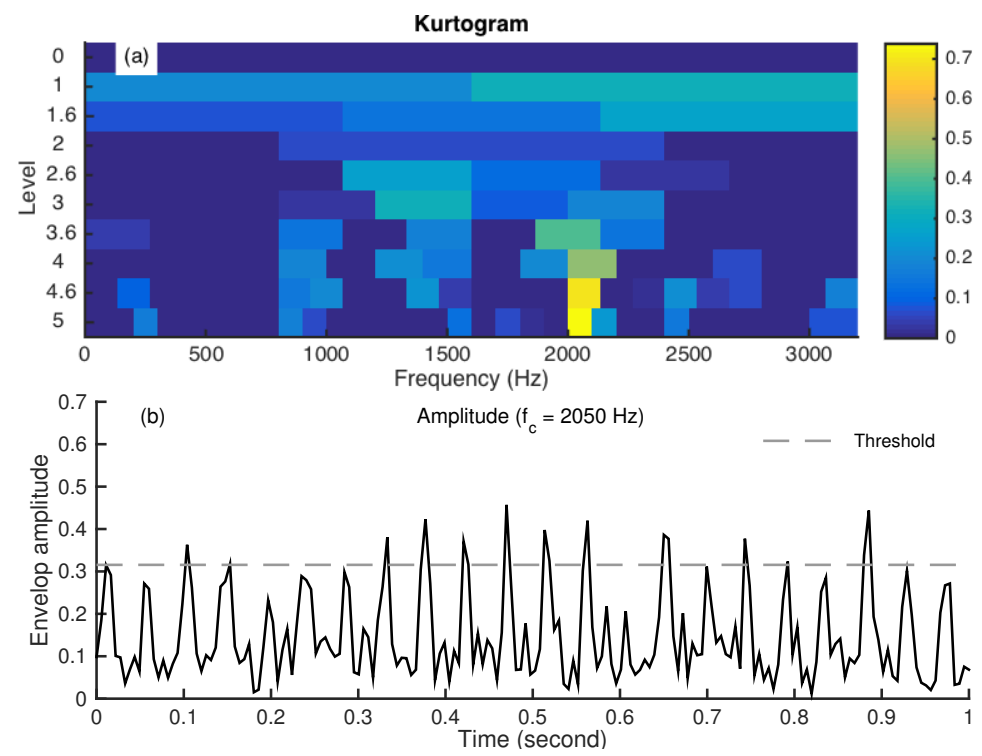

Figure 15: Example 3: Results of fast spectral kurtosis. (a) Kurtogram. (b) Envelope of extracted transients. 
To further demonstrate the effectiveness of the proposed approach, we also processed the vibration signal using spectral kurtosis and the results are presented in Fig. 15. Fig. 15(a) is the fast kurtogram, where the optimal carrier is detected at $2050 \mathrm{~Hz}$. Under this frequency, periodic transients can be observed in the envelope of the filtered signal, in accordance with the rotating speed of motor $(23.89 \mathrm{~Hz})$, as shown in Fig 15(b). No further fault-related information can be observed in Fig. 15.(b).

\section{Conclusion}

This paper proposes a periodic group sparsity approach for the purpose of detecting faults in rotating machinery. The approach uses non-convex penalty functions to promote periodic group sparsity. We show how to constrain the non-convex penalty functions to ensure that the objective function is convex. The OGS method was introduced in [9] and extended to non-convex regularized OGS in [10]. A novelty of the proposed approach is that the proposed penalty function models the periodicity of the sparse groups, making it suitable specifically for feature extraction in machinery fault diagnosis. The period of the sparse pulses is chosen based on prior knowledge of machine geometry under inspect. Moreover, the proposed approach is able to separate compound fault features by utilizing different periods of the periodic pulses corresponding to different fault frequencies (e.g., outer race and inner race characteristic frequencies of rolling element bearings) as demonstrated in Section 5 . The effectiveness of the proposed approach is verified by simulation and experimental data. The processed results demonstrate that the proposed approach outperforms other methods.

\section{Appendices}

\section{A Proof of Proposition 1}

Proof. This proposition can be proven by taking the second-order derivative, where $\phi(v ; a)$ is twice differentiable when $v \neq 0$. The second-order derivative of 12 is

$$
p^{\prime \prime}(v)=\gamma+\lambda \phi^{\prime \prime}(v ; a), \quad v \neq 0 .
$$

Therefore, when $v \neq 0$, it is sufficient that, if $\gamma+\lambda \phi^{\prime \prime}(v ; a)>0$, then $p^{\prime \prime}(v)>0$. When $v=0$, Lemma A in 10] can be utilized directly, to show that, since $p^{\prime}\left(0^{-}\right)<p^{\prime}\left(0^{+}\right)$, under the condition (13), function $p$ in 12 is strictly convex on $\mathbb{R}$.

\section{B Proof of Proposition 2}

Proof. We rewrite $\sum_{k} b_{k} u_{k}^{2}$ with the respect to 10 as

$$
\sum_{k \in \mathcal{K}} b_{k} u_{k}^{2}=\sum_{k \in \mathcal{K}_{1}} b_{k} u_{k}^{2}+\sum_{k \in \mathcal{K}_{0}} b_{k} u_{k}^{2}
$$

Then 18 is given by

$$
P(u)=\frac{1}{2 K_{1}}[\sum_{k \in \mathcal{K}_{1}} b_{k} u_{k}^{2}+\underbrace{\sum_{k \in \mathcal{K}_{0}} b_{k} u_{k}^{2}}_{0}]+\lambda \phi\left([\sum_{k \in \mathcal{K}_{1}} b_{k} u_{k}^{2}+\underbrace{\sum_{k \in \mathcal{K}_{0}} b_{k} u_{k}^{2}}_{0}]^{1 / 2} ; a\right)
$$




$$
=\frac{1}{2 K_{1}} \sum_{k \in \mathcal{K}_{1}} u_{k}^{2}+\lambda \phi\left(\left[\sum_{k \in \mathcal{K}_{1}} u_{k}^{2}\right]^{1 / 2} ; a\right) .
$$

Using Proposition 1 with $v=\left[\sum_{k \in \mathcal{K}_{1}} u_{k}^{2}\right]^{1 / 2}$ and $\gamma=1 / K_{1}$, it follows that, if $[19)$ is satisfied, then $P$ is strictly convex.

\section{References}

[1] A. Heng, S. Zhang, A. C. Tan, J. Mathew, Rotating machinery prognostics: State of the art, challenges and opportunities, Mech. Syst. Signal Process. 23 (3) (2009) 724-739.

[2] R. Yan, R. X. Gao, X. Chen, Wavelets for fault diagnosis of rotary machines: A review with applications, Signal Process. 96 (2014) 1-15.

[3] R. B. Randall, J. Antoni, Rolling element bearing diagnostics - a tutorial, Mech. Syst. Signal Process. 25 (2) (2011) 485-520.

[4] F. Cong, J. Chen, G. Dong, F. Zhao, Short-time matrix series based singular value decomposition for rolling bearing fault diagnosis, Mech. Syst. Signal Process. 34 (1) (2013) 218-230.

[5] Y. Lei, J. Lin, Z. He, M. J. Zuo, A review on empirical mode decomposition in fault diagnosis of rotating machinery, Mech. Syst. Signal Process. 35 (1) (2013) 108-126.

[6] C. Zhang, B. Li, B. Chen, H. Cao, Y. Zi, Z. He, Periodic impulsive fault feature extraction of rotating machinery using dual-tree rational dilation complex wavelet transform, ASME J. Manuf. Sci. Eng. 136 (5) (2014) 051011.

[7] R. Yan, R. X. Gao, Harmonic wavelet-based data filtering for enhanced machine defect identification, J. Sound Vib. 329 (15) (2010) 3203-3217.

[8] W. He, Y. Zi, B. Chen, F. Wu, Z. He, Automatic fault feature extraction of mechanical anomaly on induction motor bearing using ensemble super-wavelet transform, Mech. Syst. Signal Process. 54-55 (2015) 457-480.

[9] P.-Y. Chen, I. W. Selesnick, Translation-invariant shrinkage/thresholding of group sparse signals, Signal Processing 94 (2014) 476-489.

[10] P.-Y. Chen, I. W. Selesnick, Group-sparse signal denoising: Non-convex regularization, convex optimization, IEEE Trans. Signal Process. 62 (13) (2014) 3464-3478.

[11] Q. He, X. Wang, Time-frequency manifold correlation matching for periodic fault identification in rotating machines, J. Sound Vib. 332 (10) (2013) 2611-2626.

[12] B. Chen, Z. Zhang, C. Sun, B. Li, Y. Zi, Z. He, Fault feature extraction of gearbox by using overcomplete rational dilation discrete wavelet transform on signals measured from vibration sensors, Mech. Syst. Signal Process. 33 (2012) 275-298.

[13] Z. Li, Z. He, Y. Zi, Y. Wang, Customized wavelet denoising using intra-and inter-scale dependency for bearing fault detection, J. Sound Vib. 313 (1) (2008) 342-359. 
[14] W. He, Y. Zi, B. Chen, S. Wang, Z. He, Tunable Q-factor wavelet transform denoising with neighboring coefficients and its application to rotating machinery fault diagnosis, Sci. China Technol. Sci. 56 (8) (2013) 1956-1965.

[15] I. Selesnick, I. Bayram, Sparse signal estimation by maximally sparse convex optimization, IEEE Trans. Signal Process. 62 (5) (2014) 1078-1092.

[16] K. Lange, Optimization, Springer New York, 2004.

[17] S. Boyd, L. Vandenberghe, Convex optimization, Cambridge university press, 2009.

[18] I. W. Selesnick, A. Parekh, I. Bayram, Convex 1-d total variation denoising with non-convex regularization, IEEE Signal Processing Letters 22 (2) (2015) 141-144.

[19] M. Figueiredo, J. Bioucas-Dias, R. Nowak, Majorization-minimization algorithms for wavelet-based image restoration, IEEE Trans. Image Process. 16 (12) (2007) 2980-2991.

[20] R. R. Coifman, D. L. Donoho, Translation-invariant de-noising, in: Wavelet and statistics, SpringerVerlag, 1995, pp. 125-150.

[21] J. Antoni, R. Randall, The spectral kurtosis: application to the vibratory surveillance and diagnostics of rotating machines, Mech. Syst. Signal Process. 20 (2) (2006) 308-331.

[22] S. Lee, P. White, Higher-order time-frequency analysis and its application to fault detection in rotating machinery, Mech. Syst. Signal Process. 11 (4) (1997) 637-650.

[23] J. Nichols, S. Trickey, M. Seaver, S. Motley, Using ROC curves to assess the efficacy of several detectors of damage-induced nonlinearities in a bolted composite structure, Mech. Syst. Signal Process. 22 (7) (2008) 1610-1622.

[24] D. Donoho, I. Johnstone, I. M. Johnstone, Ideal spatial adaptation by wavelet shrinkage, Biometrika 81 (1993) 425-455.

[25] H. Endo, R. Randall, Enhancement of autoregressive model based gear tooth fault detection technique by the use of minimum entropy deconvolution filter, Mechanical Systems and Signal Processing 21 (2) (2007) 906-919. 